\title{
The prognostic value of pretreatment prognostic nutritional index in patients with small cell lung cancer and it's influencing factors: a meta-analysis of observational studies
}

\author{
Ai-Min Jiang ${ }^{1} \wedge$, Rui Zhao $^{2}, \mathrm{Na} \mathrm{Liu}^{1}$, Yu-Yan Ma ${ }^{1}$, Meng-Di Ren ${ }^{1}$, Tao Tian ${ }^{1}$, Yu Yao ${ }^{1}$ \\ ${ }^{1}$ Department of Medical Oncology, The First Affiliated Hospital of Xi'an Jiaotong University, Xi'an, China; ${ }^{2}$ Department of Nutrition and Food \\ Hygiene, School of Public Health, Tongji Medical College, Huazhong University of Science and Technology, Wuhan, China \\ Contributions: (I) Conception and design: Y Yao, T Tian; (II) Administrative support: Y Yao; (III) Provision of study materials or patients: AM Jiang, \\ R Zhao; (IV) Collection and assembly of data: AM Jiang, YY Ma, MD Ren; (V) Data analysis and interpretation: AM Jiang, R Zhao, N Liu; (VI) \\ Manuscript writing: All authors; (VII) Final approval of manuscript: All authors. \\ Correspondence to: Yu Yao; Tao Tian. Department of Medical Oncology, The First Affiliated Hospital of Xi'an Jiaotong University, No. 277 Yanta \\ West Road, Xi’an 710061, China. Email: 13572101611@163.com; tiantao0607@163.com.
}

\begin{abstract}
Background: Numerous studies identified that pretreatment prognostic nutritional index (PNI) was significantly associated with the prognosis in various kinds of malignant tumors. However, the prognostic value of PNI in small cell lung cancer (SCLC) remains controversial. We performed the present metaanalysis to estimate the prognostic value of PNI in SCLC and to explore the relationship between PNI and clinical characteristics.
\end{abstract}

Methods: We systematically and comprehensively searched PubMed, EMBASE, and Web of Science for available studies until April 17, 2020. Pooled hazard ratios (HRs) and their 95\% confidence intervals (CIs) were used to evaluate the correlation between PNI and overall survival (OS) and progression-free survival (PFS) in SCLC. Odds ratios (ORs) and 95\% CIs were applied to evaluate the relationship between clinical features and PNI in SCLC.

Results: A total of nine studies with 4,164 SCLC patients were included in the meta-analysis. The pooled data elucidated that lower PNI status was an independent risk factor for worse OS in SCLC (HR =1.43; 95\% CI: 1.24-1.64; $\mathrm{P}<0.001$ ), while there was no significant correlation between PNI status and PFS (HR =1.44; 95\% CI: 0.89-2.31; P=0.134). We also found that Eastern Cooperative Oncology Group (ECOG) performance status $\geq 2(\mathrm{OR}=2.72 ; 95 \% \mathrm{CI}: 1.63-4.53 ; \mathrm{P}<0.001)$ and extensive-stage $(\mathrm{ES})$ disease $(\mathrm{OR}=1.93$; 95\% CI: 1.62-2.30; $\mathrm{P}<0.001)$ were risk factors for low PNI, while prophylactic cranial irradiation (PCI) (OR $=0.53 ; 95 \%$ CI: $0.40-0.69 ; \mathrm{P}<0.001)$ was a protective factor for low PNI.

Conclusions: Our findings suggested that low PNI status was closely correlated with the decreased OS in SCLC. Surveillance on PNI, amelioration of nutritional and immune status, and timely initiation of PCI may improve the prognosis of SCLC.

Keywords: Prognostic nutritional index (PNI); small cell lung cancer (SCLC); prognosis; meta-analysis

Submitted Apr 23, 2020. Accepted for publication Aug 28, 2020.

doi: $10.21037 /$ jtd-20-1739

View this article at: http://dx.doi.org/10.21037/jtd-20-1739

^ ORCID: 0000-0002-4092-342X. 


\section{Introduction}

Lung cancer is the most common malignant tumor type and the predominant cause of cancer-related deaths worldwide (1). The 5-year survival rate is only approximately $17 \%$ for lung cancer (2). Although small cell lung cancer (SCLC) only accounts for $15 \%$ of the pathological types of lung cancer (3), it is usually characterized by highly aggressive, early distant metastasis, and genomic instability, with an overall 5-year survival rate of less than $8 \%(4,5)$. Even though an early response to chemotherapy and radiotherapy is apparent for SCLC patients, they are predisposed to early recurrence and widespread metastasis, and most patients already have metastatic dissemination at the time of diagnosis, with worrisome prognosis $(6,7)$. Therefore, it is necessary and vital to find appropriate prognostic biomarkers to effectively predict the prognosis of SCLC, which will be of great significance in improving the survival rate and implementing individual and precise management for these patients.

The prognostic nutritional index (PNI) was initially mentioned in 1980, and it was used to reflect the nutritional and immune status of patients by calculating the serum albumin level and total lymphocyte count in peripheral blood $(8,9)$. Mounting evidence has shown that low PNI status is associated with unfavorable prognosis in gastrointestinal cancer (10-14), genitourinary cancer $(15,16)$, gynecological cancer $(17,18)$, and nasopharyngeal carcinoma $(19,20)$. Recently, several studies revealed that PNI has a potential prognostic value in non-small cell lung cancer (NSCLC) $(9,21,22)$. However, there was no consistent conclusion of whether PNI could be used as a potential prognostic biomarker in patients with SCLC. Hence, we presented the following article to estimate the prognostic value of PNI in SCLC patients and to analyze the relationship between PNI and clinical characteristics in these individuals in accordance with the Primary Reporting Items for Systematic Reviews and Meta-Analyses (PRISMA) reporting checklist (23) (available at http://dx.doi. org/10.21037/jtd-20-1739).

\section{Methods}

\section{Search strategy}

The present meta-analysis was performed according to the PRISMA statement. We systematically and comprehensively searched PubMed, EMBASE, and Web of Science to determine the available literature. The retrieval time was from database establishment to April 17, 2020. The following search terms were used for study filtration: ("the prognostic nutritional index" OR "PNI") AND ("lung cancer" OR "lung tumor" OR "lung carcinoma" OR "lung neoplasm" OR “small cell lung cancer" OR "SCLC"). Besides, to obtain potential eligible studies, we also manually searched pertinent references cited in the identified articles. This meta-analysis was registered in PROSPERO (http:// www.crd.york.ac.uk/PROSPERO) and the registration number for this article is CRD42020192407.

\section{Eligibility criteria}

Studies were considered as eligible based on the following criteria: (I) patients were diagnosed with SCLC through histopathological or cytological confirmation; (II) the PNI value was evaluated before treatment; (III) the correlations between PNI and overall survival (OS) or progression-free survival (PFS) were reported in the identified studies; (IV) hazard ratios (HRs) and their 95\% confidence intervals (CIs) were available in the multivariate analysis; (V) case-control or cohort studies. Studies were excluded if they were published as reviews, conference abstracts, letters, and case reports. We also excluded articles not published in English.

\section{Data extraction}

Two investigators (AMJ and RZ) extracted the data into Excel according to standardized formats independently. Any discrepancies regarding data extraction between them were resolved by discussion and consulting with another investigator (NL) for a consensus. The extracted data mainly included (I) basic characteristics of the included studies (first author of the study, year of publication, country, and sample size); (II) clinical characteristics of the included subjects (gender, age, disease stage, treatment type, PNI cut-off value, methods of cut-off value determination, median follow up time, and methods of survival analysis); (III) HR and its corresponding 95\% CI between PNI and OS or PFS in multivariate analysis.

\section{Quality assessment}

The Newcastle-Ottawa Scale (NOS) was applied to quality assessment for included studies in our meta-analysis (24). The score for each study was determined from study selection, comparability assessment, determination of exposure and outcome, with a total score varies from 0 to 9. 


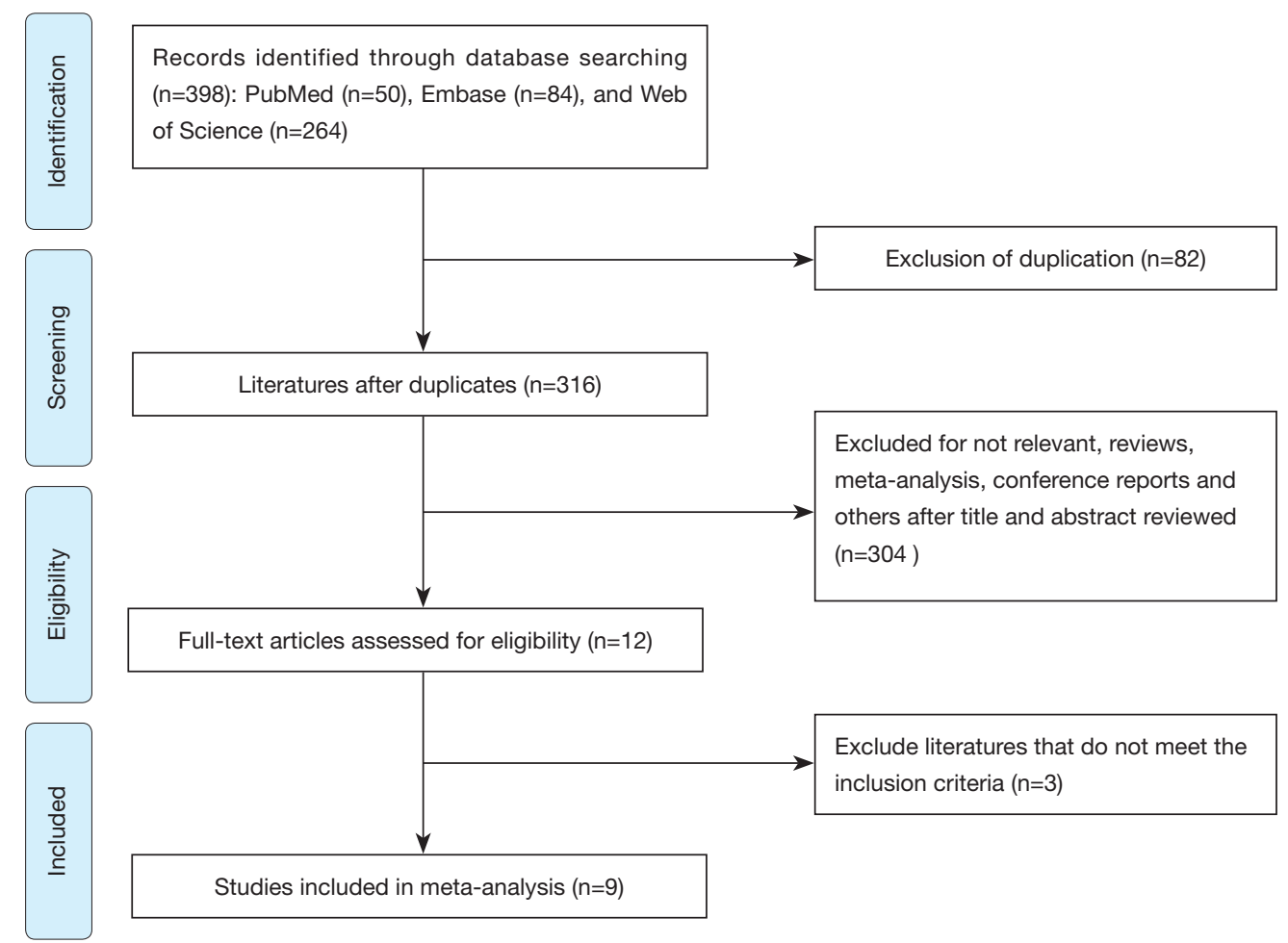

Figure 1 Flow chart of literature selection.

Studies with a score of not less than seven were considered high-quality studies. Two investigators (YYM and MDR) conducted quality assessment independently.

\section{Statistical analysis}

All statistical analyses were performed using STATA version 12.0 (Stata Corporation, College Station, Texas, USA) in our study. The pooled HRs and $95 \%$ CIs were calculated to estimate the correlation between PNI and the prognosis of SCLC. Odds ratios (ORs) and 95\% CIs were applied to evaluate the relationship between clinical features and PNI of SCLC. Cochran's Q test and $\mathrm{I}^{2}$ test were used to assess the statistical heterogeneity among the included studies, with significant statistical heterogeneity considered as $\mathrm{I}^{2}>50 \%$ and $\mathrm{P} \leq 0.10$. A random-effect model was adopted, and subgroup analyses were performed to explore the potential sources of heterogeneity when significant statistical heterogeneity was detected. Otherwise, a fixedeffect model was adopted for pooled data analysis. We used sensitivity analysis to assess the stability of the pooled HRs by excluding each study sequentially from the meta-analysis. We adopted Begg's and Egger's tests to detect publication bias.

\section{Results}

\section{Study selection and study characteristics}

After a comprehensive and systematic search from electronic databases, we identified 398 potentially relevant studies. We remained 316 studies after removing duplicated literature. Subsequently, we screened titles and abstracts carefully, 304 studies were excluded, including irrelevant studies, reviews, conference abstracts, and others. After reading the full text, we excluded three studies that did not meet the inclusion criteria. Ultimately, a total of nine studies with 4,164 SCLC patients were included in the present meta-analysis. The detailed process of literature selection was presented in Figure 1.

Table 1 presented the detailed characteristics of the included studies. All the included literature were retrospective studies and published between 2015 and 2020. Of these, six studies were conducted in China (4,7,25,28-30), and the rest three studies were conducted in Japan (26), South Korea (27), and Turkey (31). Among these, six studies $(4,7,25,27,28,31)$ enrolled SCLC patients with limitedstage (LS)/extensive-stage (ES) disease, two studies $(26,29)$ enrolled patients with ES disease, and only one study (30) 


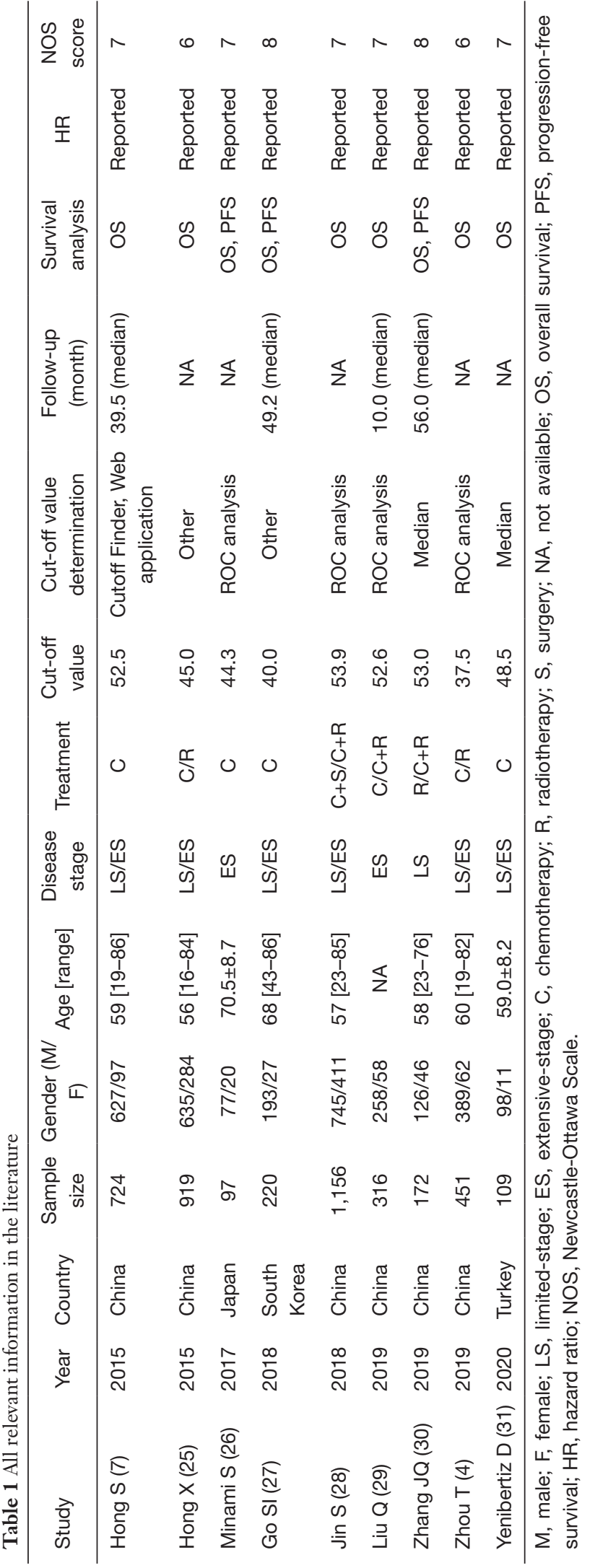

focused on patients with LS disease. The majority of patients were male $(3,148,75.6 \%)$, and the age of the subjects was ranged from 16 to 86 years old. The median sample size was 316 for the included studies (range, $97-1,156)$, and the median PNI cut-off value was 48.5 (range, 37.5-53.9). There were seven high-quality studies after performing quality assessment (Table 2).

\section{Correlation between PNI and OS in SCLC}

A total of nine studies reported HRs and 95\% CIs between OS and PNI in SCLC. As the results of the heterogeneity test indicated significant heterogeneity among the studies $\left(\mathrm{I}^{2}=66.8 \%, \mathrm{P}=0.002\right)$, we applied a random effect model for pooled data analysis. The result revealed that low PNI was an independent risk factor for worse OS in SCLC (HR =1.43; 95\% CI: 1.24-1.64; $\mathrm{P}<0.001$, Figure 2). Subsequently, we performed subgroup analyses stratified by country, sample size, tumor stage, treatment type, PNI cut-off value, the methods of cut-off value determination, and NOS score to explore the potential sources of heterogeneity. The results demonstrated that the heterogeneity was significantly reduced after stratified by sample size and tumor stage, while there was still significant heterogeneity across the remaining subgroups (Table 3). Therefore, the sample size and tumor stage might be the potential sources of heterogeneity. Besides, the subgroup analyses stratified by the methods of PNI cut-off value determination revealed that low PNI was associated with the worse OS when the cut-off value was determined by ROC curve analysis (HR $=1.48 ; 95 \%$ CI: 1.17-1.87; $\mathrm{P}=0.001)$ and other methods (HR $=1.37 ; 95 \%$ CI: 1.09-1.72; $\mathrm{P}=0.007)$. However, low PNI was not significantly correlated with the OS in SCLC when the median was used to determine the PNI cut-off value $(\mathrm{HR}=1.45$; 95\% CI: 0.86-2.43; $\mathrm{P}=0.160$ ). Moreover, the results of other subgroups confirmed that low PNI was significantly correlated with unfavorable OS in SCLC, as summarized in Table 3.

\section{Correlation between PNI and PFS in SCLC}

There were three studies reported HRs and 95\% CIs between PFS and PNI in SCLC. Since the heterogeneity test suggested that there was significant heterogeneity among the included studies $\left(\mathrm{I}^{2}=63.3 \%, \mathrm{P}=0.066\right)$, we adopted a random effect model to calculate the pooled data. The result of the pooled data analysis revealed that there was no significant correlation between low PNI and PFS in SCLC (HR =1.44; 95\% CI: 0.89-2.31; P=0.134, Figure 3). 
Table 2 Quality assessment conducted according to the NOS for all included studies

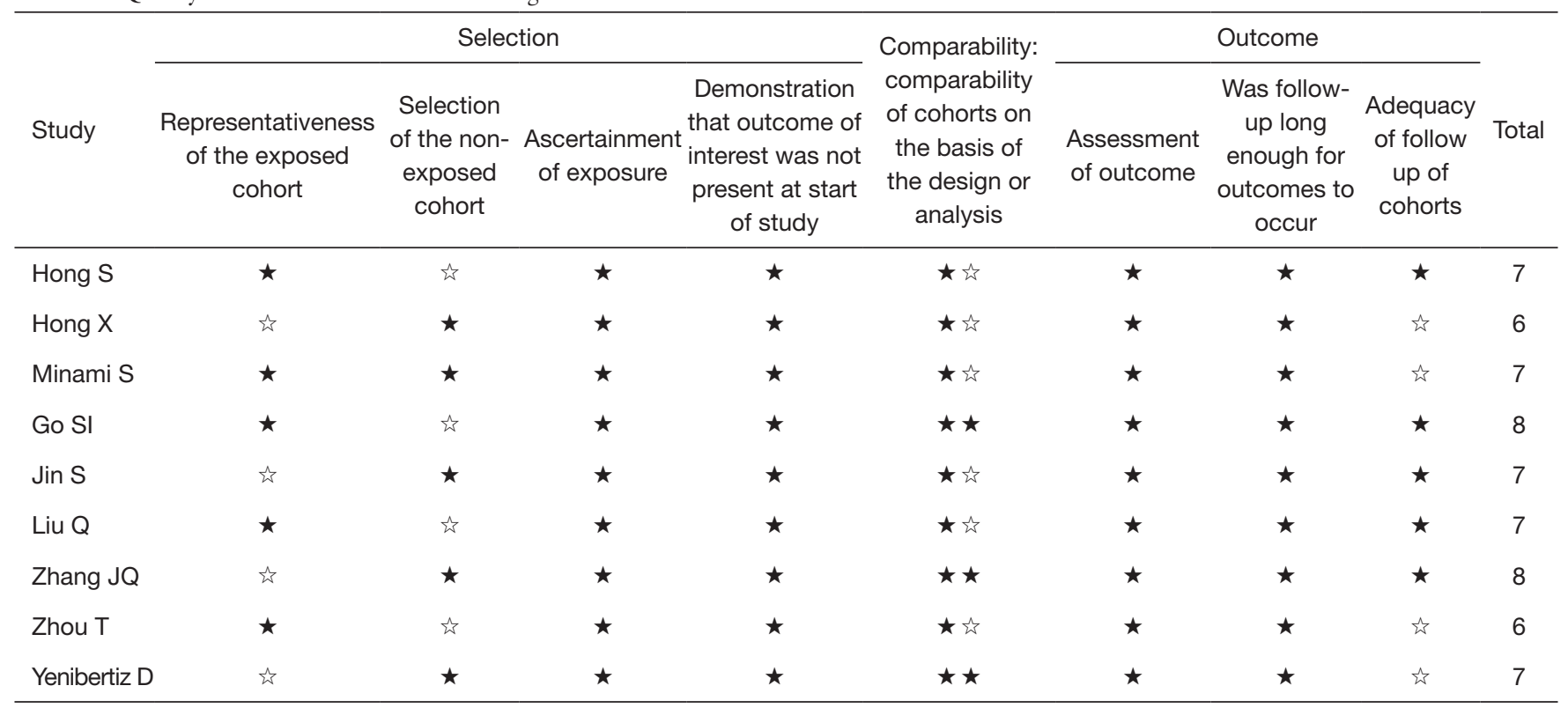

$\star$, represents points of score; is , means no score. NOS, Newcastle-Ottawa Scale.

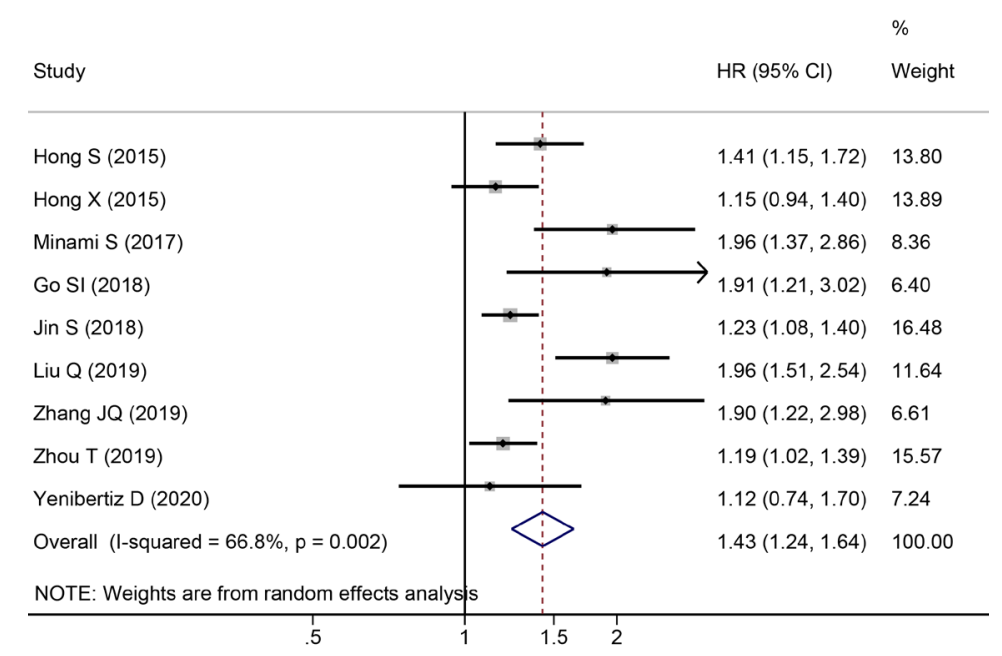

Figure 2 Forest plot of the association between low PNI status and OS in patients with SCLC. PNI, prognostic nutritional index; OS, overall survival; SCLC, small cell lung cancer.

\section{Correlation between PNI and clinical characteristics in SCLC}

To explore the risk factors of low PNI in SCLC, we further analyzed the relationship between low PNI status and clinical characteristics of the enrolled patients in each eligible study. The analyzed clinical characteristics mainly included gender (male $v s$. female), smoking history (smoker vs. never smoker), Eastern Cooperative Oncology Group (ECOG) performance status (2-3 vs. 0-1), disease stage (ES vs. LS), and whether received prophylactic cranial irradiation (PCI) (PCI vs. non-PCI). We found that ECOG performance status $\geq 2$ (OR $=2.72$; 95\% CI: $1.63-4.53$; $\mathrm{P}<0.001$, Figure $4 A)$ and $\mathrm{ES}$ disease $(\mathrm{OR}=1.93 ; 95 \% \mathrm{CI}$ : $1.62-2.30 ; \mathrm{P}<0.001$, Figure $4 B$ ) were risk factors for low PNI. However, PCI was a protective factor for low PNI in 
Table 3 Subgroup analyses for low PNI status on OS in SCLC patients

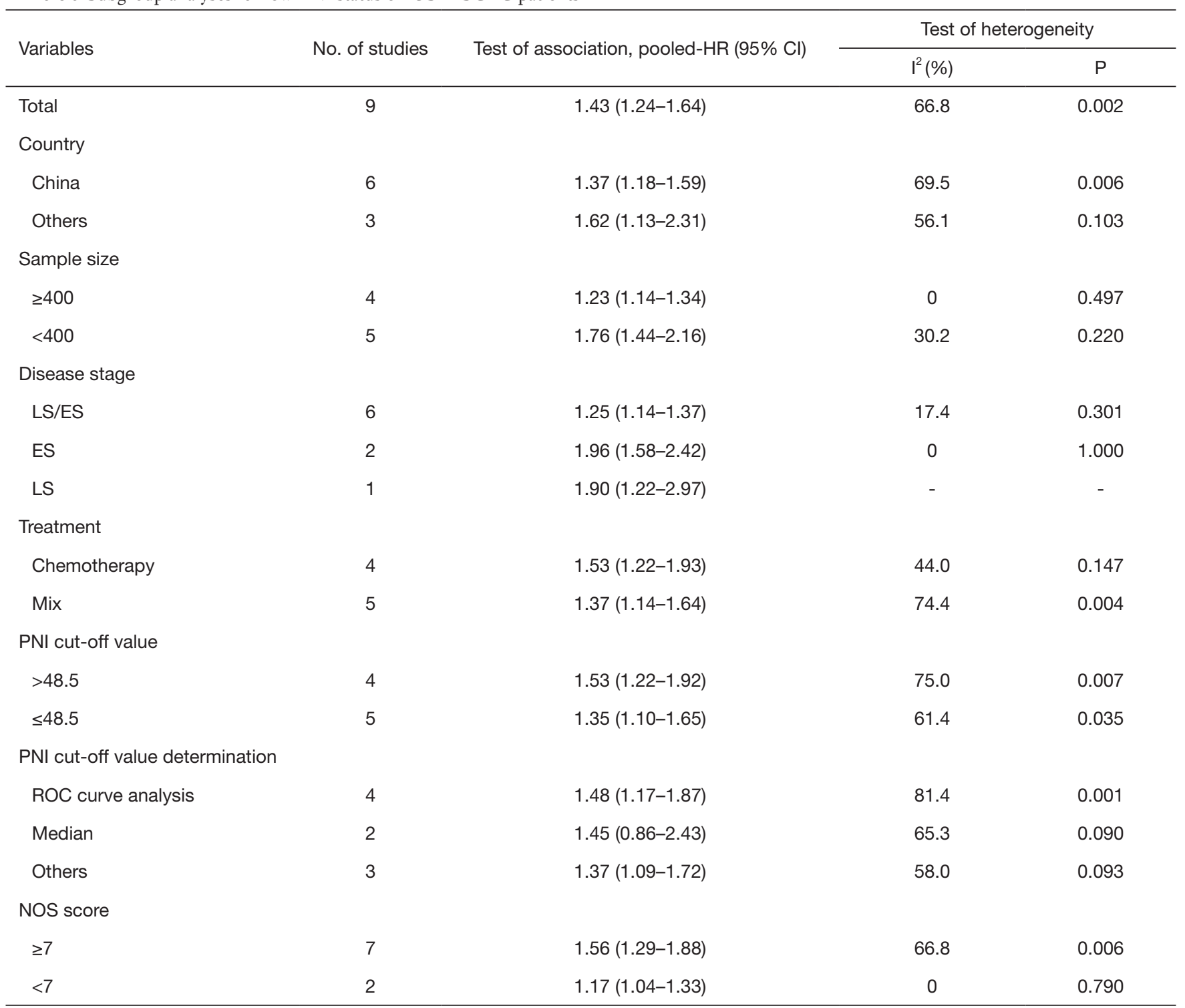

PNI, prognostic nutritional index; OS, overall survival; SCLC, small cell lung cancer; LS, limited-stage; ES, extensive-stage; NOS, Newcastle-Ottawa Scale.

SCLC patients $(\mathrm{OR}=0.53 ; 95 \%$ CI: $0.40-0.69 ; \mathrm{P}<0.001$, Figure 4C). Besides, it showed that gender ( $\mathrm{OR}=1.04 ; 95 \%$ CI: $0.78-1.37 ; \mathrm{P}=0.798$, Figure $4 D$ ) and smoking history ( $\mathrm{OR}=1.10 ; 95 \%$ CI: 0.74-1.65; $\mathrm{P}=0.631$, Figure $4 E$ ) were not significantly correlated with the occurrence of low PNI.

\section{Sensitivity analysis and publication bias}

To evaluate the stability of the pooled data, we performed sensitivity analysis by omitting each study sequentially from the meta-analysis. It showed that the pooled HRs for OS fluctuated between the pooled $95 \%$ CIs, indicating the pooled HRs for OS in SCLC were stable (Figure 5). Subsequently, to detect the existence of publication bias, we performed Begg's test and Egger's test. Begg's funnel plot showed good symmetry (Figure 6), indicating that there was no significant publication bias. Further quantitative analyses revealed that the literature included in the present study did not exist publication bias (Begg's test: $\mathrm{P}=0.175$, Egger's test: $\mathrm{P}=0.254)$. Because of limited data are available for low 


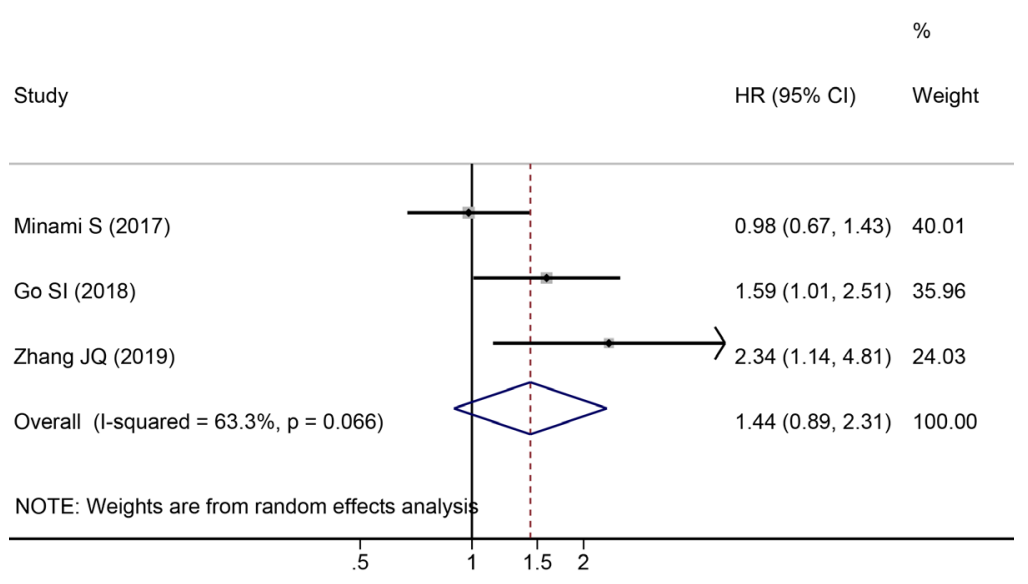

Figure 3 Forest plot of the association between low PNI status and PFS in patients with SCLC. PNI, prognostic nutritional index; PFS, progression-free survival; SCLC, small cell lung cancer.

PNI and PFS in the included studies, we did not perform sensitivity analysis and publication bias test.

\section{Discussion}

SCLC is considered as a lethal and highly aggressive malignant tumor due to its characteristics of rapid tumor growth, early recurrence, and widespread metastasis (32). Although significant improvements have been seen in early detection and treatment in SCLC, it remains a worse prognosis (32). There is an urgent need to find a potential biomarker that can effectively predict the prognosis of SCLC to improve the clinical outcome. In recent decades, numerous studies identified that PNI status before treatment was significantly associated with the survival outcomes in various malignant tumors. Previously published meta-analyses also showed that low PNI was closely related with worse OS $(9,21,22)$ and PFS $(21,22)$ in NSCLC. Although there is an increasing number of studies reported that PNI was also related to the prognosis in SCLC, these results derived from different centers, with controversial conclusions. Therefore, we conducted this study to evaluate the prognostic value of PNI in SCLC via meta-analysis, and to explore the relationship between PNI and clinical characteristics of these individuals.

A total of nine studies with 4,164 SCLC patients were included in the current meta-analysis. The result indicated that low PNI status before treatment was significantly associated with a reduced OS in SCLC. Consistent with the pooled result, the results of subgroup analyses showed that low PNI was also significantly associated with worse OS in
SCLC when the studies were stratified by country, sample size, disease stage, treatment type, PNI cut-off value, and NOS score. However, subgroup analysis stratified by the methods of PNI cut-off value determination showed that low PNI was associated with the worse OS when the cut-off value was determined by ROC curve analysis and other methods, while low PNI was not significantly associated with the OS in SCLC when the median was used to determine the PNI cut-off value. On the one hand, it may be attributed to the fact that only two studies used the median to determine the PNI cut-off value in the include studies. On the other hand, it also suggests that the appropriate methods should be applied to determine the cut-off value in future studies. Further sensitivity analysis and publication bias test showed that the pooled data with good robustness. Therefore, PNI can be significant in predicting the OS in SCLC. However, the result of our study showed that PNI status was not significantly associated with the PFS in SCLC. Considering only three studies reported the data for PFS, it needs to be further validated in the future.

PNI is a widely used nutritional indicator that can reflect the nutritional and immune status of patients with malignancy based on the serum albumin level and total lymphocyte count in peripheral blood (9). Several potential mechanisms can explain the relationship between low PNI and poor prognosis in SCLC. First of all, the serum albumin level in PNI can significantly reflect the nutritional status of patients. Previous studies reported that hypoproteinemia was frequently related to reduced quality of life and diminished life expectancy due to 
A
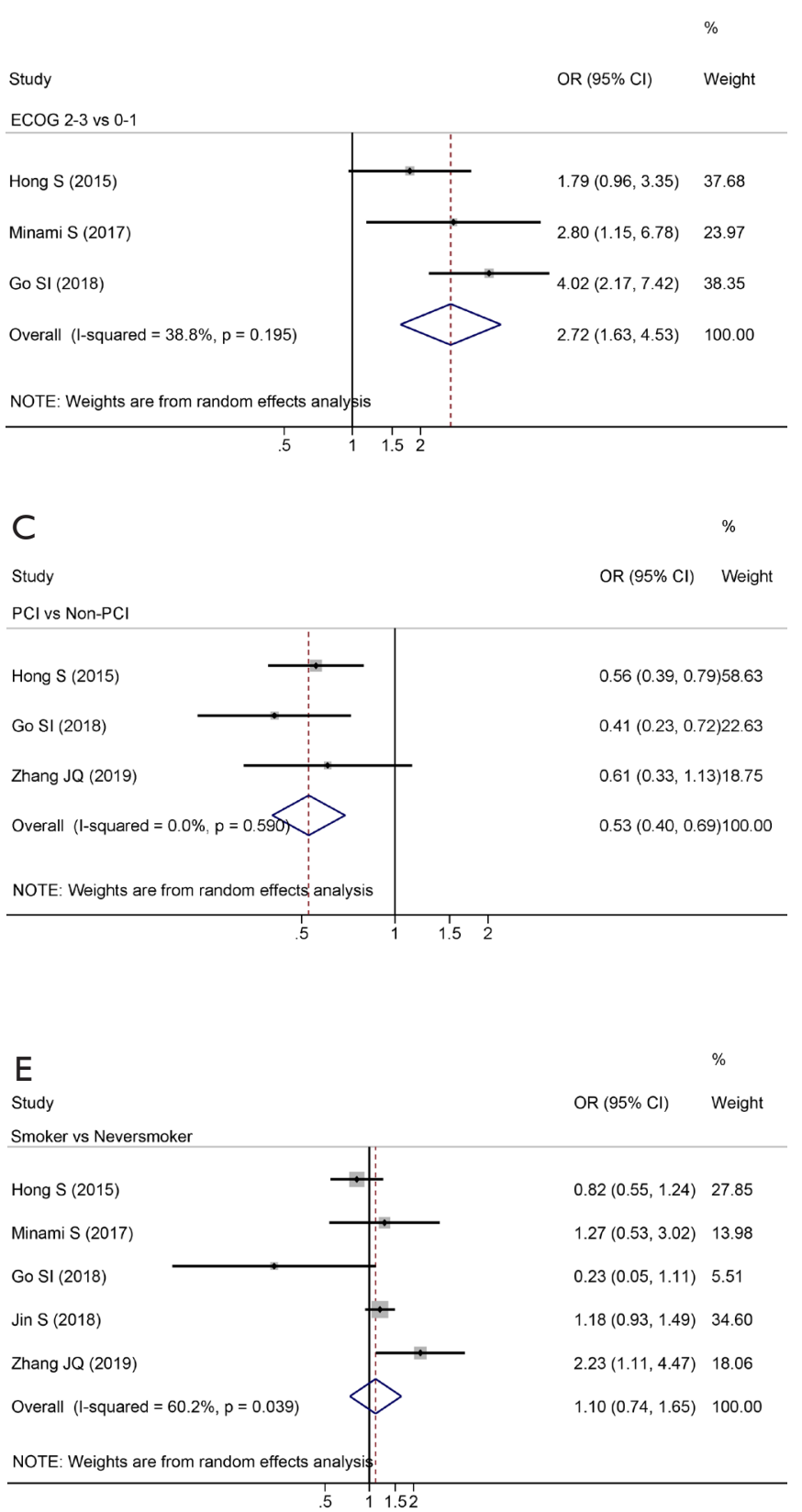

B

Study
ES vs LS

D

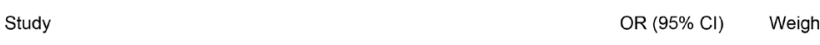

Male vs Female

Weight

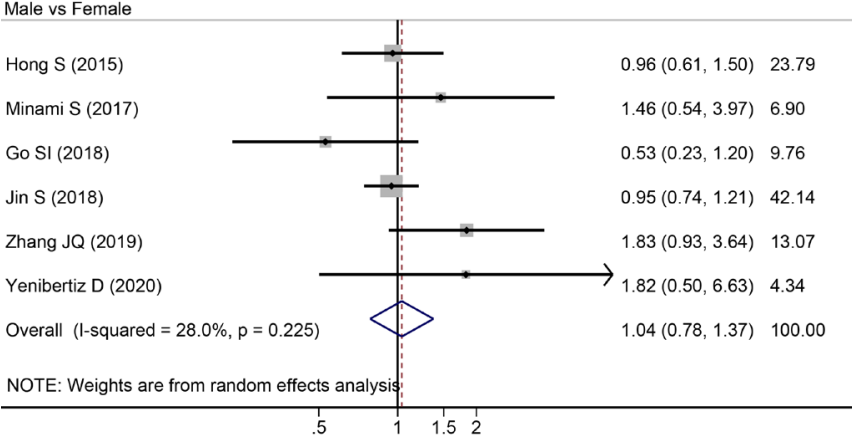

Figure 4 Forest plot of the association between low PNI status and clinical characteristics of patients with SCLC. (A) ECOG performance status, (B) disease stage, (C) PCI, (D) gender, (E) smoking histology. PNI, prognostic nutritional index; SCLC, small cell lung cancer; ECOG, Eastern Cooperative Oncology Group; PCI, prophylactic cranial irradiation.

immunosuppression and diminished muscle mass in patients with malignancy $(33,34)$. Furthermore, Paccagnella et al. also reported that hypoproteinemia in cancer patients could result in malnutrition and weight loss, thus leading to a poor prognosis and raised cancer-associated deaths in these patients $(35,36)$. Moreover, in recent years, the importance of inflammation and the immune system has been highlighted in numerous studies (37-39). On the one hand, inflammation within the tumor microenvironment is closely related to cancer development and progression 


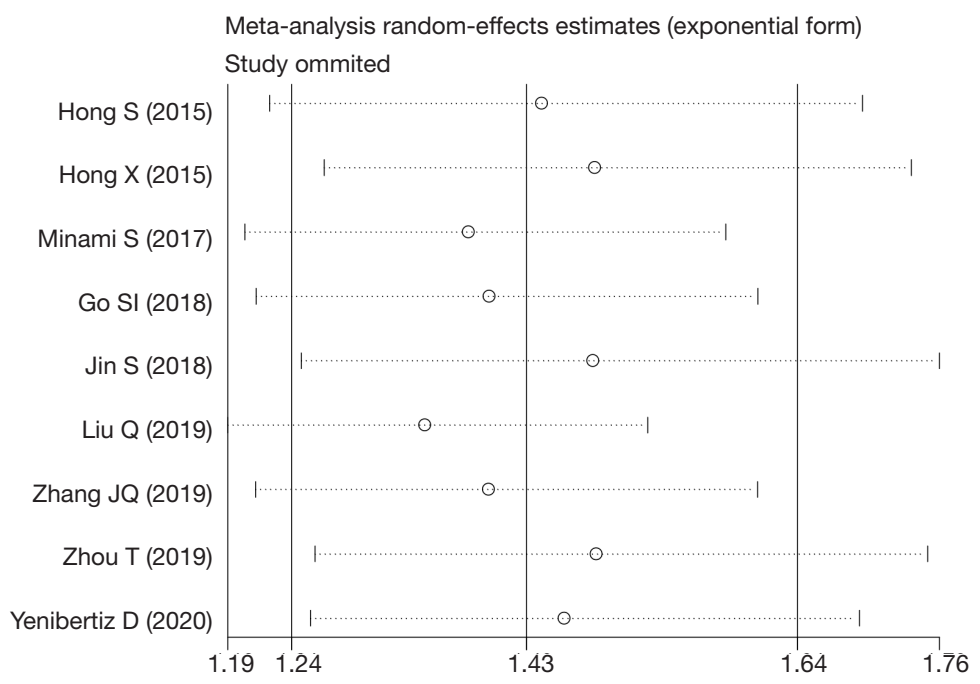

Figure 5 Sensitivity analysis of the relationship between low PNI status and OS in patients with SCLC. PNI, prognostic nutritional index; OS, overall survival; SCLC, small cell lung cancer.

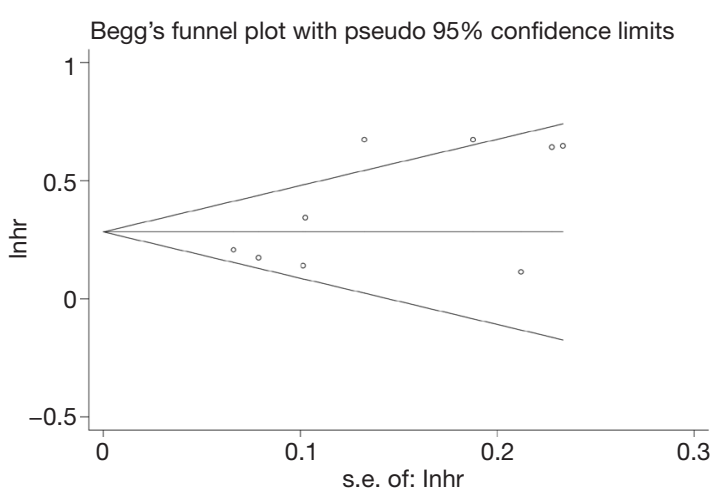

Figure 6 Funnel plot of publication bias for OS in patients with SCLC. OS, overall survival; SCLC, small cell lung cancer.

due to various mechanisms (37). On the other hand, lymphocyte in peripheral blood can reflect the systemic inflammatory state of patients with malignancy, and it plays a crucial role in cell-mediated immune response (9). A recent retrospective study conducted in Japan also reported that lymphocytopenia was associated with worse OS in LSSCLC (39). Taken together, PNI is a significant prognostic factor in SCLC.

We then explored the relationship between PNI status and clinical features of SCLC patients. It revealed that ECOG performance status $\geq 2$ and ES disease were risk factors for the occurrence of low PNI. However, PCI was a protective factor for the occurrence of low PNI. It can be explained by the fact that patients with worse ECOG performance status and ES disease are frequently associated with malnutrition, cachexia, and impaired immune response. Besides, Suzuki et al. reported that LS-SCLC patients who received PCI were correlated with favorable OS and higher total lymphocyte count (39).

To the best of our knowledge, our study is the first metaanalysis that comprehensively evaluated the prognostic value of PNI in SCLC and explored the relationship between PNI and clinical characteristics in these patients. However, several limitations in the present study need to be noticed. First of all, there was significant heterogeneity in our pooled analysis, and the results of subgroup analyses revealed that sample size and disease stage might be the potential sources of heterogeneity. Furthermore, the majority of included studies were conducted in Asia, with retrospective design, lacking prospective studies and data from other regions. Moreover, considering only three studies focused on the prognostic value of PNI for PFS in SCLC, the correlation between PNI and PFS needs to be further validated. Therefore, large-scale, multicenter, and well-designed prospective studies are needed to validate our results.

\section{Conclusions}

In summary, PNI can be significant in predicting the prognosis in SCLC. The current meta-analysis also suggested that low PNI was associated with ECOG performance status, disease stage, and PCI. Surveillance 
on PNI, amelioration of nutritional and immune status, and timely initiation of PCI may improve the prognosis of patients with SCLC. More large-scale and multicenter prospective studies are warranted to validate our results.

\section{Acknowledgments}

Funding: None.

\section{Footnote}

Reporting Checklist: The authors have completed the PRISMA reporting checklist. Available at http://dx.doi. org/10.21037/jtd-20-1739

Conflicts of Interest: All authors have completed the ICMJE uniform disclosure form (available at http://dx.doi. org/10.21037/jtd-20-1739). The authors have no conflicts of interest to declare.

Ethical Statement: The authors are accountable for all aspects of the work in ensuring that questions related to the accuracy or integrity of any part of the work are appropriately investigated and resolved.

Open Access Statement: This is an Open Access article distributed in accordance with the Creative Commons Attribution-NonCommercial-NoDerivs 4.0 International License (CC BY-NC-ND 4.0), which permits the noncommercial replication and distribution of the article with the strict proviso that no changes or edits are made and the original work is properly cited (including links to both the formal publication through the relevant DOI and the license). See: https://creativecommons.org/licenses/by-nc-nd/4.0/.

\section{References}

1. Chen Z, Fillmore CM, Hammerman PS, et al. Non-smallcell lung cancers: a heterogeneous set of diseases. Nat Rev Cancer 2014;14:535-46.

2. Chen $W$, Zheng R, Baade PD, et al. Cancer statistics in China, 2015. CA Cancer J Clin 2016;66:115-32.

3. Byers LA, Rudin CM. Small cell lung cancer: Where do we go from here? Cancer 2015;121:664-72.

4. Zhou T, Zhao Y, Zhao S, et al. Comparison of the prognostic value of systemic inflammation response markers in small cell lung cancer patients. J Cancer 2019;10:1685-92.
5. Sabari JK, Lok BH, Laird JH, et al. Unravelling the biology of SCLC: implications for therapy. Nat Rev Clin Oncol 2017;14:549-61.

6. Shepherd FA, Crowley J, Van Houtte P, et al. The International Association for the Study of Lung Cancer Lung Cancer Staging Project: Proposals Regarding the Clinical Staging of Small Cell Lung Cancer in the Forthcoming (Seventh) Edition of the Tumor, Node, Metastasis Classification for Lung Cancer. J Thorac Oncol 2007;2:1067-77.

7. Hong $S$, Zhou T, Fang $W$, et al. The prognostic nutritional index (PNI) predicts overall survival of small-cell lung cancer patients. Tumour Biol 2015;36:3389-97.

8. Buzby GP, Mullen JL, Matthews DC, et al. Prognostic nutritional index in gastrointestinal surgery. Am J Surg 1980;139:160-7.

9. Li D, Yuan X, Liu J, et al. Prognostic value of prognostic nutritional index in lung cancer: a meta-analysis. J Thorac Dis 2018;10:5298-307.

10. Schwegler I, von Holzen A, Gutzwiller JP, et al. Nutritional risk is a clinical predictor of postoperative mortality and morbidity in surgery for colorectal cancer. Br J Surg 2010;97:92-7.

11. Nazha B, Moussaly E, Zaarour M, et al. Hypoalbuminemia in colorectal cancer prognosis: Nutritional marker or inflammatory surrogate? World J Gastrointest Surg 2015;7:370-7.

12. Nakatani M, Migita K, Matsumoto S, et al. Prognostic significance of the prognostic nutritional index in esophageal cancer patients undergoing neoadjuvant chemotherapy. Dis Esophagus 2017;30:1-7.

13. Saito H, Kono Y, Murakami Y, et al. Influence of prognostic nutritional index and tumor markers on survival in gastric cancer surgery patients. Langenbecks Arch Surg 2017;402:501-7.

14. Lee SH, Chung MJ, Kim B, et al. The Significance of the Prognostic Nutritional Index for All Stages of Pancreatic Cancer. Nutr Cancer 2017;69:512-9.

15. Fan L, Wang $\mathrm{X}$, Chi $\mathrm{C}$, et al. Prognostic nutritional index predicts initial response to treatment and prognosis in metastatic castration-resistant prostate cancer patients treated with abiraterone. Prostate 2017;77:1233-41.

16. Peng D, Gong YQ, Hao H, et al. Preoperative Prognostic Nutritional Index is a Significant Predictor of Survival with Bladder Cancer after Radical Cystectomy: a retrospective study. BMC Cancer 2017;17:391.

17. Zhang W, Ye B, Liang W, et al. Preoperative prognostic nutritional index is a powerful predictor of prognosis in patients with stage III ovarian cancer. Sci Rep 2017;7:9548. 
18. Haraga J, Nakamura K, Omichi C, et al. Pretreatment prognostic nutritional index is a significant predictor of prognosis in patients with cervical cancer treated with concurrent chemoradiotherapy. Mol Clin Oncol 2016;5:567-74.

19. Miao J, Xiao W, Wang L, et al. The value of the Prognostic Nutritional Index (PNI) in predicting outcomes and guiding the treatment strategy of nasopharyngeal carcinoma (NPC) patients receiving intensity-modulated radiotherapy (IMRT) with or without chemotherapy. J Cancer Res Clin Oncol 2017;143:1263-73.

20. Du XJ, Tang LL, Mao YP, et al. Value of the prognostic nutritional index and weight loss in predicting metastasis and long-term mortality in nasopharyngeal carcinoma. J Transl Med 2015;13:364.

21. Hu Y, Shen J, Liu R, et al. Prognostic value of pretreatment prognostic nutritional index in non-small cell lung cancer: A systematic review and meta-analysis. Int J Biol Markers 2018;33:372-8.

22. Wang Z, Wang Y, Zhang X, et al. Pretreatment prognostic nutritional index as a prognostic factor in lung cancer: Review and meta-analysis. Clin Chim Acta 2018;486:303-10.

23. Knobloch K, Yoon U, Vogt PM. Preferred reporting items for systematic reviews and meta-analyses (PRISMA) statement and publication bias. J Craniomaxillofac Surg 2011;39:91-2.

24. Stang A. Critical evaluation of the Newcastle-Ottawa scale for the assessment of the quality of nonrandomized studies in meta-analyses. Eur J Epidemiol 2010;25:603-5.

25. Hong X, Cui B, Wang M, et al. Systemic Immuneinflammation Index, Based on Platelet Counts and Neutrophil-Lymphocyte Ratio, Is Useful for Predicting Prognosis in Small Cell Lung Cancer. Tohoku J Exp Med 2015;236:297-304.

26. Minami S, Ogata Y, Ihara S, et al. Pretreatment Glasgow prognostic score and prognostic nutritional index predict overall survival of patients with advanced small cell lung cancer. Lung Cancer (Auckl) 2017;8:249-57.

27. Go SI, Jeon H, Park SW, et al. Low pre-treatment nutritional index is significantly related to poor outcomes in small cell lung cancer. Thorac Cancer 2018;9:1483-91.

28. Jin S, Cao S, Xu S, et al. Clinical impact of pretreatment prognostic nutritional index (PNI) in small cell lung cancer patients treated with platinum-based chemotherapy. Clin Respir J 2018;12:2433-40.

29. Liu Q, Yang W. Relationship between systemic immuneinflammation index and prognosis of patients with brain metastases from small cell lung cancer. Tumor 2019;39:649-57.

30. Zhang JQ, Wang YY, Xu KP, et al. Prognostic evaluation of nutritional indicators in patients with limited-stage small cell lung cancer. Zhonghua Zhong Liu Za Zhi 2019;41:937-42.

31. Yenibertiz D, Ozyurek BA, Erdogan Y. Is Onodera's prognostic nutritional index (OPNI) a prognostic factor in small cell lung cancer (SCLC)? Clin Respir J 2020. [Epub ahead of print].

32. Gazdar AF, Bunn PA, Minna JD. Small-cell lung cancer: what we know, what we need to know and the path forward. Nat Rev Cancer 2017;17:725-37.

33. Soeters PB, Wolfe RR, Shenkin A. Hypoalbuminemia: Pathogenesis and Clinical Significance. JPEN J Parenter Enteral Nutr 2019;43:181-93.

34. Gupta D, Lis CG. Pretreatment serum albumin as a predictor of cancer survival: a systematic review of the epidemiological literature. Nutr J 2010;9:69.

35. Paccagnella A, Morassutti I, Rosti G. Nutritional intervention for improving treatment tolerance in cancer patients. Curr Opin Oncol 2011;23:322-30.

36. Fruchtenicht AVG, Poziomyck AK, Kabke GB, et al. Nutritional risk assessment in critically ill cancer patients: systematic review. Revista Brasileira de terapia Intensiva 2015;27:274-83.

37. Elinav E, Nowarski R, Thaiss CA, et al. Inflammationinduced cancer: crosstalk between tumours, immune cells and microorganisms. Nat Rev Cancer 2013;13:759-71.

38. Templeton AJ, McNamara MG, Seruga B, et al. Prognostic role of neutrophil-to-lymphocyte ratio in solid tumors: a systematic review and meta-analysis. J Natl Cancer Inst 2014;106:dju124.

39. Suzuki R, Wei X, Allen PK, et al. Prognostic Significance of Total Lymphocyte Count, Neutrophilto-lymphocyte Ratio, and Platelet-to-lymphocyte Ratio in Limited-stage Small-cell Lung Cancer. Clin Lung Cancer 2019;20:117-23.

Cite this article as: Jiang AM, Zhao R, Liu N, Ma YY, Ren MD, Tian T, Yao Y. The prognostic value of pretreatment prognostic nutritional index in patients with small cell lung cancer and it's influencing factors: a meta-analysis of observational studies. J Thorac Dis 2020;12(10):5718-5728. doi: $10.21037 /$ jtd-20-1739 